\title{
Aplicações de metadados baseadas em FRBR e RDA em repositórios institucionais digitais: uma revisão sistemática da literatura
}

\author{
FRBR and RDA based metadata applications \\ in digital institutional repositories: a \\ systematic review of literature
}

\author{
Natalia Gallo CERRAO' (iD) 0000-0002-8450-3451 \\ Fabiano Ferreira de CASTRO' 1 (D) 0000-0002-8712-2654
}

\section{Resumo}

Em função das transformações tecnológicas no campo da Catalogação Descritiva, em especial no domínio bibliográfico, com o desenvolvimento de modelos conceituais, como os Functional Requirements for Bibliographic Records (Requisitos Funcionais para Registros Bibliográficos) e a proposta das orientações Resource Description and Access (Descrição de Recursos e Acesso) para a descrição de recursos, faz-se necessário compreender de que maneira essas iniciativas se relacionam com os repositórios institucionais digitais. O objetivo desta pesquisa foi o estudo dos Functional Requirements for Bibliographic Records e das Resource Description and Access estabelecidos no domínio bibliográfico, a fim de identificar o estado da arte sobre as tendências do domínio bibliográfico e a possibilidade de aplicá-los a repositórios institucionais digitais. O aporte teórico e metodológico da Revisão Sistemática da Literatura propiciou o mapeamento e a identificação de elementos-chave para responder à questão de pesquisa. Foram analisados vinte e cinco documentos na Revisão Sistemática da Literatura, o que pode ser considerado um índice relativamente baixo sobre a produção científica condizente à proposta de pesquisa e à necessidade de estudos mais aprofundados acerca do tema. Foram identificadas tecnologias computacionais, casos de uso e aplicações específicas em ambientes bibliográficos, acadêmicos e na Web. Sublinha-se que o estado da arte construído se apresenta como um ponto de partida para o desenvolvimento de ferramentas e de aplicações que aproximem o domínio bibliográfico dos repositórios institucionais digitais.

Palavras-chave: Descrição de Recursos e Acesso. Domínio bibliográfico. Metadados. Repositórios institucionais. Requisitos Funcionais para Registros Bibliográficos.

\section{Abstract}

Given the technological transformations in the field of Descriptive Cataloging, especially in the bibliographic domain, and the development of conceptual models, such as the Functional Requirements for Bibliographic Records and the proposal of the Resource

\footnotetext{
1 Universidade Federal de São Carlos, Programa de Pós-Graduação em Ciência da Informação, Grupo de Pesquisas e Estudos em Representação do Conhecimento e Tecnologias da Informação e Comunicação. Caixa Postal 676, Brasil. Correspondência para/Correspondence to: F. F. CASTRO: E-mail: $<$ fabianocastro@ufscar.br>.

Artigo baseado na dissertação de N. G. CERRAO, intitulada "Análise das aplicações de metadados baseada em FRBR e RDA em repositórios institucionais digitais: contribuições do domínio bibliográfico". Universidade Federal de São Carlos, São Carlos, 2019.

Recebido em 21 de outubro de 2019, reapresentado em 4 de maio de 2020 e aprovado em 8 de julho de 2020.

Como citar este artigo/How to cite this article

Cerrao, N. G.; Castro, F. F. Aplicações de metadados baseadas em FRBR e RDA em repositórios institucionais digitais: uma revisão sistemática da literatura. Transinformação, v. 32, e190080, 2020. https://doi.org/10.1590/2318-0889202032e190080
} 
Description and Access guidelines for the description of resources, it is necessary to understand how such initiatives relate to digital institutional repositories. The objective of this research was to study the Functional Requirements for Bibliographic Records and Resource Description and Access established in the bibliographic domain, in order to identify the state of the art of the currents trends of the bibliographic domain and if they can be applied to digital institutional repositories. The theoretical and methodological support of the Systematic Literature Review provided the mapping and identification of the key elements to answer the research question. Twenty five documents were analyzed in the Systematic Literature Review, which can be considered a relatively low index on the scientific production consistent with the research proposal, pointing to the need for further studies on the subject. Computational technologies, use cases, and specific applications were identified in bibliographic, academic, and web-based environments. The state of the art provided in this research constitutes a starting point for the development of tools and applications that bring the bibliographic domain closer to digital institutional repositories.

Keywords: Resource Description and Access. Bibliographic domain. Metadata. Functional Requirements for Bibliographic Records. Institutional repositories.

\section{Introdução}

Os processos de trabalho e as estruturas de armazenamento da informação passam por transfigurações em meio ao progresso cientíico e ao crescente avanço do acesso à Internet. Isso pode ser observado em função da adaptação da sociedade às tecnologias disponíveis, em especial às Tecnologias da Informação e Comunicação (TIC).

Pesquisadores da área da Ciência da Informação sempre se preocuparam com o desenvolvimento e com o aperfeiçoamento de padrões e de modelos de estrutura e de conteúdo que permitam maior qualidade e confiabilidade na representação, no armazenamento e na posterior disseminação da informação, de forma a estudar os conceitos, os procedimentos e os métodos de descrição que garantam sua adequada recuperação.

Destacam-se, nesta pesquisa, as tendências do domínio bibliográfico para o tratamento descritivo da informação. Entre elas, os Functional Requirements for Bibliographic Records (FRBR, Requisitos Funcionais para Registros Bibliográficos), um modelo conceitual baseado na modelagem entidade-relacionamento que facilita a organização de registros bibliográficos e possibilita a construção e a modelagem de catálogos mais funcionais, otimizando os processos de busca e de recuperação da informação. Também são ressaltadas as orientações Resource Description and Access (RDA, Descrição de Recursos e Acesso), as quais consistem em uma iniciativa para a orientação da descrição de recursos, proposta em meio à atualização dos esquemas e dos códigos de catalogação.

Visto que o modelo conceitual FRBR e as orientações RDA foram construídos para contemplar recursos tanto analógicos quanto - e principalmente - digitais, procurou-se compreendê-los no contexto dos Repositórios Institucionais Digitais (RI) e do esquema de metadados Dublin Core.

Os repositórios institucionais são ambientes informacionais destinados a reunir, a armazenar, a gerenciar e a disseminar a produção científica de uma instituição. Ressalta-se que grande parte dos repositórios digitais tem sido construída com base no padrão de metadados Dublin Core (Castro; Santos, 2011).

Dessa maneira, esta pesquisa se propõe a analisar em que medida o modelo conceitual FRBR e as orientações RDA podem ser aplicados em repositórios institucionais digitais.

O método utilizado foi a Revisão Sistemática da Literatura (RSL), a qual oportunizou a identificação de modelos de aplicação de metadados baseados em FRBR e em RDA em repositórios institucionais digitais.

\section{Representação de recursos informacionais no domínio bibliográfico}

Conforme Alves (2016), a partir do início do século XXI, o domínio bibliográfico passa por uma grande reestruturação dos métodos de representação da informação, devido à integração das tecnologias disponíveis à atividade da catalogação descritiva. 
Neste cenário surgem novos princípios; modelos conceituais que reestruturam as tarefas realizadas no domínio e as estruturas de representação; novos códigos de catalogação e, consequentemente, inicia-se o desenvolvimento de propostas para padrões bibliográficos mais flexíveis e compatíveis com a estrutura da Web (Alves, 2016, p. 101).

Nesta pesquisa, destacam-se o modelo conceitual FRBR e as orientações RDA como algumas das principais iniciativas e convites à reflexão sobre os impactos tecnológicos na Catalogação Descritiva.

\section{Requisitos Funcionais para Registros Bibliográficos}

Ao longo de oito anos, pesquisadores da International Federation of Library Associations and Institutions (IFLA, Federação Internacional de Associações e Instituições Bibliotecárias) desenvolveram os FRBR e o relatório final foi publicado em 1998 (Moreno; Arellano, 2005).

Os Functional Requirements for Bibliographic Records propõem um modelo conceitual para catálogos bibliográficos, uma proposta comum para facilitar a organização de registros bibliográficos por meio da modelagem entidade-relacionamento, metodologia advinda da Ciência da Computação que faz a intersecção com a Ciência da Informação. Esse modelo tem o objetivo de descrever os objetos de um domínio (entidades) que possuem características (atributos) e se relacionam a outras entidades.

As entidades FRBR são divididas em três grupos. Nesta pesquisa, foram utilizadas as ponderações de Tillett (2004) e de Oliver (2011). O Grupo 1 diz respeito às entidades que são produtos de trabalho intelectual ou artístico: Obra, Expressão, Manifestação e Item. O Grupo 2 representa as responsáveis pelo conteúdo intelectual ou artístico, pela sua produção e pela sua disseminação: Pessoa e Entidade Coletiva. Apesar de não estar definida nos FRBR, a "Família" é, também, considerada uma entidade do Grupo 2 (International ..., 2016). As do Grupo 3 representam o conjunto de temas que expressam diferentes assuntos de uma obra: Conceito, Objeto, Evento e Lugar. Também podem ser incluídas nesse grupo as entidades dos Grupos 1 e 2.

Cada uma das entidades definidas no modelo FRBR é associada a um conjunto de características ou atributos. "Os atributos da entidade servem como meio pelos quais os usuários formulam consultas e interpretam respostas quando buscam informações sobre uma entidade particular" (International ..., 2009, p. 31, tradução nossa)².

Os relacionamentos servem como veículo para representar as ligações entre uma entidade e outra e, assim, como meio de auxiliar o usuário a "navegar" pelo universo representado em uma bibliografia, em um catálogo ou em um banco de dados bibliográfico (International ..., 2009).

Além dos modelos conceituais desenvolvidos para a construção de catálogos bibliográficos, faz-se necessário o aprimoramento de regras e de esquemas de descrição que possibilitem a convergência ao ambiente digital.

Nesse sentido, a integração dos FRBR numa proposta de criação de um código de catalogação que substitua o Anglo-American Cataloguing Rules (AACR2r, Código de Catalogação Anglo-Americano) culminou na criação e no desenvolvimento das orientações Resource Description and Access (RDA), uma tendência no contexto de iniciativas de criação de códigos de catalogação.

\section{Resource Description and Access}

As tendências da Catalogação Descritiva são diretamente influenciadas por regras estabelecidas pela comunidade de domínio, estruturadas nos esquemas de descrição e nos códigos de catalogação. Esses códigos

\footnotetext{
$\overline{2}$ No original: "The attributes of the entity serve as the means by which users formulate queries and interpret responses when seeking information about a particular entity".
} 
padronizam o processo de representação da informação nos aspectos que envolvem a forma e o conteúdo de um recurso informacional.

Percebe-se um investimento na Catalogação Descritiva, em uma tentativa de se criar modelos e estruturas capazes de atender às exigências da descrição de recursos no contexto digital e às necessidades dos usuários. Uma dessas propostas consiste nas RDA, tendência no desenvolvimento de códigos de catalogação.

As Resource Description and Access, inicialmente projetado com a nomenclatura AACR3, foi criado com o objetivo de revisar os princípios do AACR2r e de ser o seu sucessor (RDA ..., 2018). Além disso, possui diretrizes e instruções que abrangem a descrição e o acesso dos recursos digitais e analógicos, resultando em registros que podem ser usados em uma variedade de ambientes digitais (Internet, OPACs [On-line Public Access Catalog] da Web etc.).

De acordo com Oliver (2011), o objetivo das RDA é facilitar a descrição de recursos seguindo um processo de decisão lógica, tratando-se de um padrão projetado para ser de fácil utilização e para gerar registros que contenham dados relevantes e importantes para os usuários.

As Resource Description and Access analisa dados em registros bibliográficos na perspectiva de como são utilizados e apresenta um modelo conceitual (FRBR), o qual identifica as entidades no universo bibliográfico de interesse do usuário (obras, itens, pessoas etc.), os atributos dessas entidades e suas relações entre si.

Atualmente, a adoção das RDA, de forma que permita o uso de todo o seu potencial, não constitui uma simples escolha administrativa. Para se atingir esse objetivo, são necessários, previamente, o planejamento, a modelagem e a construção de catálogos que possam refletir a base entidade-atributo-relacionamento presente nos modelos conceituais FRBR e FRAD (Functional Requirements for Authority Data) (Assumpção; Santos, 2013).

É essencial compreender como as ferramentas, os instrumentos, os conceitos, os métodos, as tecnologias e os processos advindos da Catalogação Descritiva podem e poderão atuar e ressignificar a forma de descrição dos dados e dos recursos nos ambientes informacionais, no domínio bibliográfico. Desse modo, o método de Revisão Sistemática da Literatura possibilitou a identificação de elementos teóricos e metodológicos para a proposta de análise de aplicações de metadados em repositórios institucionais digitais baseados em FRBR e em RDA.

\section{Caminhos metodológicos: Revisão Sistemática da Literatura}

A Revisão Sistemática da Literatura (RSL) se caracteriza como um método de revisão da literatura acrescida de etapas e de critérios rigorosos, garantindo à pesquisa científica maior confiabilidade e reprodutibilidade.

Uma revisão sistemática aborda uma questão específica, utiliza métodos explícitos e transparentes para realizar uma pesquisa detalhada de literatura e avaliação crítica de estudos individuais, e desenha conclusões sobre o que atualmente conhecemos e não conhecemos sobre um determinado tópico ou questão (Briner; Denyer, 2012, p. 112, tradução nossa) ${ }^{\mathbf{3}}$.

A definição da questão de pesquisa irá traçar toda a sua continuidade, definindo seu campo e sua população e possibilitando a definição de seu protocolo. Este, incorporando as questões da revisão, deve ser elaborado antes da condução da pesquisa e garantirá que a revisão seja sistemática, transparente e replicável.

O protocolo inclui as bases de dados a serem consultadas, as palavras-chave a serem utilizadas, os critérios de inclusão e de exclusão de trabalhos e os dados a serem extraídos dos documentos selecionados.

\footnotetext{
3 No original: "A systematic review addresses a specific question, utilizes explicit and transparent methods to perform a thorough literature search and critical appraisal of individual studies, and draws conclusions about what we currently know and do not know about a given question or topic".
} 
A Revisão Sistemática proposta nesta pesquisa foi dividida em três etapas principais. Estas são: (1) planejamento, contemplando a realização de uma revisão bibliográfica como forma de familiarização com a temática estudada e de preenchimento do protocolo de busca; (2) condução, que consistiu na busca em bases de dados e na aplicação dos critérios de inclusão e de exclusão; e (3) extração de dados.

Como ferramenta instrumental para a realização da RSL proposta, foi utilizado o software StArt (State of the Art through Systematic Review), desenvolvido pelo Laboratório de Pesquisa em Engenharia de Software (LaPES), do Departamento de Ciência da Computação da Universidade Federal de São Carlos (UFSCar) (LaPES, 2012). O apoio de uma ferramenta computacional é imprescindível para dar maior qualidade e agilidade à aplicação de uma Revisão Sistemática.

O protocolo de busca, composto no total por dezoito campos que embasam o processo de busca, de seleção e de extração de dados, foi preenchido no próprio software StArt, o qual já apresenta os campos de maneira bem definida e organizada (Quadro 1).

Quadro 1. Protocolo da Revisão Sistemática da Literatura.

\begin{tabular}{|c|c|}
\hline Campo & Preenchimento do campo \\
\hline Objective (Objetivo) & $\begin{array}{l}\text { Compreender, à luz da Revisão Sistemática da Literatura, a aplicabilidade dos modelos conceituais FRBR e } \\
\text { das orientações RDA, estabelecidos no domínio bibliográfico, em Rls. }\end{array}$ \\
\hline Main question (Questão principal) & $\begin{array}{l}\text { É possível aplicar os modelos conceituais estabelecidos no domínio bibliográfico e as orientações } \\
\text { estabelecidas pelo RDA em repositórios institucionais? }\end{array}$ \\
\hline $\begin{array}{l}\text { Population (População) } \\
\text { Intervention (Intervenção) }\end{array}$ & $\begin{array}{l}\text { Iniciativas de aplicação do modelo conceitual FRBR e das orientações RDA em repositórios institucionais. } \\
\text { Como são tratadas e discutidas as possibilidades de aplicação do modelo conceitual FRBR e das orientações } \\
\text { RDA em repositórios institucionais nos trabalhos recuperados. }\end{array}$ \\
\hline $\begin{array}{l}\text { Control (Controle) } \\
\text { Results (Resultados) }\end{array}$ & $\begin{array}{l}\text { Artigos de bases de dados da área da Ciência da Informação. } \\
\text { Identificar estudos que abordem a aplicação do modelo conceitual FRBR e das orientações RDA em } \\
\text { repositórios institucionais. Identificar possíveis requisitos para a aderência dos RIs aos modelos conceituais } \\
\text { do domínio bibliográfico e às orientações RDA. }\end{array}$ \\
\hline Application (Aplicação) & $\begin{array}{l}\text { Contribuição para a área da Ciência da Informação em relação às formas de representar a informação e } \\
\text { à modelagem de dados, aplicando, em repositórios institucionais, as novas possibilidades no âmbito da } \\
\text { Catalogação Descritiva. }\end{array}$ \\
\hline $\begin{array}{l}\text { Keywords and synonyms } \\
\text { (Palavras-chave e sinônimos) }\end{array}$ & $\begin{array}{l}\text { A string de busca utilizada em todas as bases de dados foi definida como: ("Digital repositories" OR } \\
\text { "Repositories" OR "Institutional repositories") AND ("FRBR" OR "Functional Requirements for Bibliographical } \\
\text { Records" OR "RDA"OR "Resource Description and Access"). }\end{array}$ \\
\hline $\begin{array}{l}\text { Sources selection criteria definition } \\
\text { (Definição de critérios de busca) }\end{array}$ & Trabalhos indexados em periódicos da área da Ciência da Informação. \\
\hline Studies languages (Idiomas) & Inglês; Português; Espanhol. \\
\hline $\begin{array}{l}\text { Source search methods } \\
\text { (Métodos de pesquisa) }\end{array}$ & $\begin{array}{l}\text { Leitura do título e do resumo dos documentos; aplicação dos critérios de inclusão e de exclusão; leitura da } \\
\text { introdução e da conclusão dos documentos; leitura do documento completo nos casos em que as análises } \\
\text { anteriores se mostraram inconclusivas para a seleção. }\end{array}$ \\
\hline Source list (Lista de fontes) & $\begin{array}{l}\text { As bases de dados consultadas e utilizadas foram: } \\
\text { Web of Science. } \\
\text { Library and Information Science Abstracts (LISA). } \\
\text { Library, Information Science \& Technology Abstracts with full text (LISTA). } \\
\text { Information Science \& Technology Abstracts (ISTA). }\end{array}$ \\
\hline $\begin{array}{l}\text { Study selection criteria (Inclusion } \\
\text { and Exclusion) } \\
\text { Critérios de seleção (Inclusão e } \\
\text { Exclusão) }\end{array}$ & $\begin{array}{l}\text { Os critérios de seleção foram definidos como (I) para Inclusão e (E) para Exclusão. } \\
\text { (I) Abordar a aplicação do modelo conceitual FRBR em ambientes digitais. } \\
\text { (I) Abordar aplicação das orientações das orientações RDA em ambientes digitais. } \\
\text { (I) Abordar a implementação das orientações RDA e/ou do FRBR especificamente em repositórios digitais. } \\
\text { (I) Tratar de possíveis requisitos ou modelos para a aderência à RDA e ao FRBR em ambientes ou repositórios } \\
\text { digitais. } \\
\text { (E) Documento não acessível ou não localizado integralmente. } \\
\text { (E) Não estar nos idiomas especificados. } \\
\text { (E) Não abordar a aplicação das orientações RDA em repositórios. } \\
\text { (E) Não abordar a aplicação do modelo FRBR em repositórios. }\end{array}$ \\
\hline
\end{tabular}


Quadro 1. Protocolo da Revisão Sistemática da Literatura.

\begin{tabular}{|c|c|}
\hline Campo & Preenchimento do campo \\
\hline $\begin{array}{l}\text { Studies types definition } \\
\text { (Definição de tipos de estudos) }\end{array}$ & Trabalhos indexados nas bases de dados consultadas. \\
\hline $\begin{array}{l}\text { Studies initial selection } \\
\text { (Seleção inicial) }\end{array}$ & Não se aplica à pesquisa. \\
\hline $\begin{array}{l}\text { Studies quality evaluation } \\
\text { (Avaliação de qualidade dos } \\
\text { estudos) }\end{array}$ & $\begin{array}{l}\text { Uma avaliação minuciosa da qualidade dos documentos será feita a partir da análise da metodologia dos } \\
\text { trabalhos recuperados e aceitos após a Revisão Sistemática. }\end{array}$ \\
\hline $\begin{array}{l}\text { Data extraction form fields } \\
\text { (Forma de extração de dados) }\end{array}$ & $\begin{array}{l}\text { Enfoque dos documentos; aplicação das orientações RDA em repositórios; aplicação do modelo conceitual } \\
\text { FRBR em repositórios; contribuições do domínio bibliográfico para os repositórios; considerações finais. }\end{array}$ \\
\hline $\begin{array}{l}\text { Results summarization } \\
\text { (Sumarização de resultados) }\end{array}$ & $\begin{array}{l}\text { Após extração de dados, realizada seguindo o roteiro proposto no campo "Information Extraction Fields", } \\
\text { os dados serão agrupados em quadros de acordo com a categoria de análise, os resultados semelhantes } \\
\text { serão agrupados, permitindo a realização de uma análise quantitativa e a elaboração de inferências que } \\
\text { respondam à pergunta de pesquisa. }\end{array}$ \\
\hline
\end{tabular}

Fonte: Cerrao (2019).

Nota: FRBR: Functional Requirements for Bibliographic Records; RDA: Resource Description Access.

Após definido o protocolo de busca e o seu planejamento, partiu-se para a sua execução, com buscas nas bases de dados selecionadas. Em cada uma delas utilizou-se a string ${ }^{4}$ de busca: ("Digital repositories" OR "Repositories" OR "Institutional repositories") AND ("FRBR" OR "Functional Requirements for Bibliographical Records" OR "RDA" OR "Resource Description and Access"). Nessa etapa, a quantidade total de trabalhos recuperados foi de 275 artigos. A divisão dos trabalhos recuperados por base de dados é contemplada no Quadro 2.

Após a pesquisa mencionada foram aplicados os critérios de inclusão e de exclusão, a partir de uma leitura criteriosa e objetiva da introdução, com atenção ao título, ao resumo, aos objetivos, à metodologia e à conclusão dos trabalhos identificados.

Concluída a etapa da seleção e de extração, foram aceitos 25 artigos, correspondentes a 9\% do total da amostra. 35, correspondentes a 13\% estavam duplicados. Os outros 215, equivalentes a 78\%, foram rejeitados. Os artigos duplicados (contendo mesmo título, autor e ano de publicação) foram rejeitados (Figura 1). Optou-se, para melhor visualização, pela confecção de um quadro-resumo contendo título, autor(es) e ano (de publicação) de cada artigo aceito na Revisão Sistemática da Literatura (Quadro 3).

A revisão supracitada, a partir da análise aprofundada e minuciosa dos documentos aceitos, permitiu responder se os FRBR e as orientações RDA podem ser aplicados ou adotados em repositórios institucionais digitais, e identificar casos de uso ou modelos de aplicação de metadados, assim como tecnologias informacionais e computacionais.

Quadro 2. Quantidade de trabalhos recuperados por base de dados.

\begin{tabular}{lc}
\hline Base de dados & Quantidade de Trabalhos (n) recuperados \\
\hline Web of Science & 6 \\
Library and Information Science Abstracts (LISA) & 237 \\
Library, Information Science \& Technology Abstracts with full text (LISTA) & 23 \\
Information Science \& Technology Abstracts (ISTA) & 9 \\
\hline Total & 275 \\
\hline
\end{tabular}

Fonte: Cerrao (2019).

4 Uma string de busca corresponde a uma sentença elaborada na formulação da estratégia de busca, a partir das palavras-chave estabelecidas no protocolo de revisão sistemática. 


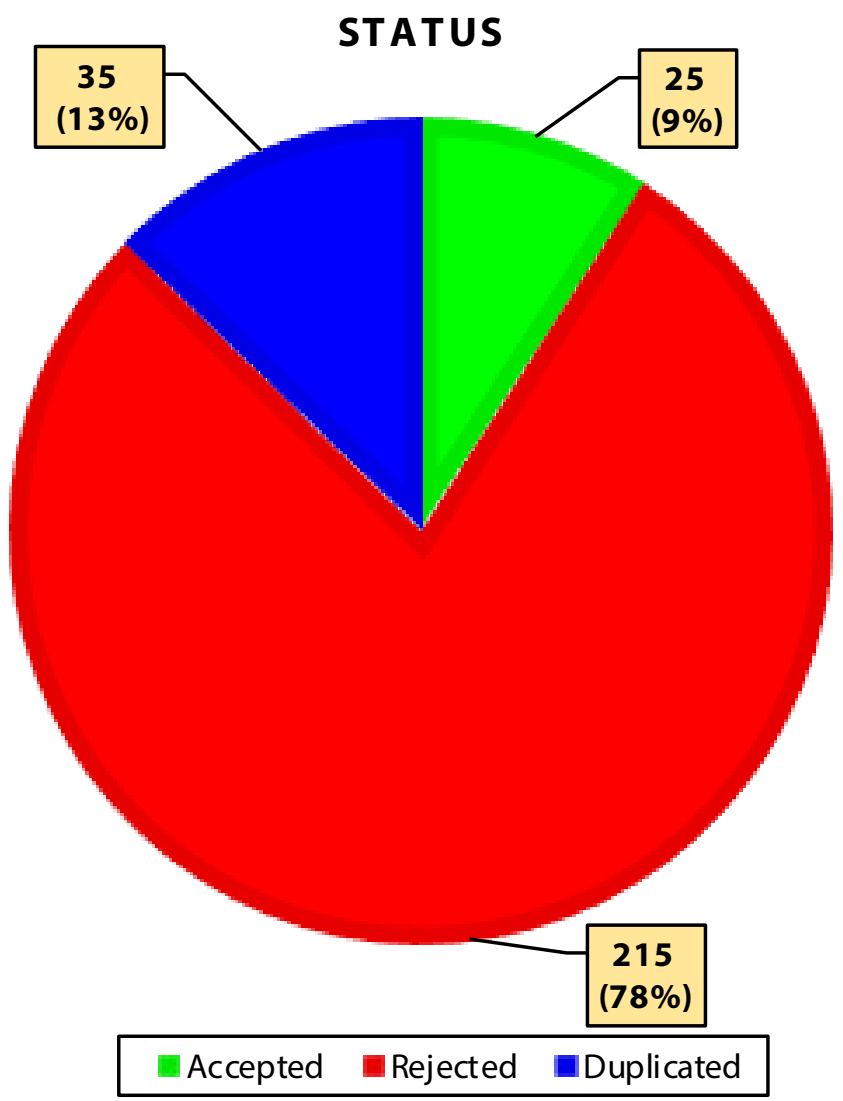

Figura 1. Resultado após a análise e seleção/extração de documentos.

Fonte: Cerrao (2019).

Quadro 3. Artigos aceitos na Revisão Sistemática da Literatura.

\begin{tabular}{|c|c|c|}
\hline Título & Autor(es) & Ano \\
\hline $\begin{array}{l}\text { Utilizing the FRBR framework in designing user-focused digital content and access } \\
\text { systems }\end{array}$ & Madison, O. M. A. & 2006 \\
\hline Understanding FRBR as a conceptual model: FRBR and the bibliographic universe & Carlyle, A. & 2006 \\
\hline Towards accessibility to digital cultural materials: a FRBRized approach & Weng, C.; Mi, J. & 2006 \\
\hline MARC, MARCXML e FRBR: relações encontradas na literatura & Moreno, F. P.; Brascher, M. & 2007 \\
\hline Changing to RDA & Oliver, C. & 2007 \\
\hline Usability study of digital institutional repositories & Kim, H. H.; Kim, Y. H. & 2008 \\
\hline Describing scholarly works with Dublin Core: a functional approach & Allinson, J. & 2008 \\
\hline Cataloging and Classification: review of the literature 2005-06 & El-Sherbini, M. & 2008 \\
\hline Assessing FRBR in Dublin Core application profiles & Chaudhri, T. & 2009 \\
\hline Metadata issues in Digital Libraries: key concepts and perspectives & Solodovnik, l. & 2011 \\
\hline $\begin{array}{l}\text { Libraries' metadata as data in the era of the Semantic Web: modeling a repository of } \\
\text { master theses and PHd dissertations for the web of data }\end{array}$ & Peponakis, M. & 2013 \\
\hline The value of MARC data, or, challenges of frbrisation & Aalberg, T; Žumer, M. & 2013 \\
\hline Experimenting with implementing FRBR in a Chinese Koha system & Chang, N.; Tsai, Y.; Dunsire, G.; Hopkinson, A. & 2013 \\
\hline $\begin{array}{l}\text { New displaying models of bibliographic data and resources: cataloguing/resource } \\
\text { description and search results }\end{array}$ & Trombone, A. & 2014 \\
\hline $\begin{array}{l}\text { Positioning Libraries for a new bibliographic universe: a review of cataloging and } \\
\text { classification literature 2011-12 }\end{array}$ & Martin, K. E.; Mundle, K. & 2014 \\
\hline Multi-entity models of resource description in the Semantic Web & Baker, T.; Coyle, K.; Petiya, S. & 2014 \\
\hline
\end{tabular}


Quadro 3. Artigos aceitos na Revisão Sistemática da Literatura.

\begin{tabular}{|c|c|c|}
\hline Título & Autor(es) & Ano \\
\hline Making search more meaningful: action values, Linked Data, and semantic relationships & Stern, D. & 2015 \\
\hline FRBR Aggregates: their types and frequency in library collections & O’Neill, E.; Žumer, M.; Mixter, J. & 2015 \\
\hline FRBR, RDA and Law Libraries & Doyle, $\mathrm{H}$. & 2015 \\
\hline FRBR, Information, and Intertextuality & Rafferty, P. & 2015 \\
\hline $\begin{array}{l}\text { Ball State University Libraries: implementing RDA for digital libraries: a collaboration } \\
\text { between cataloging and metadata librarians }\end{array}$ & Leigh, K.; Leigh, R. N. & 2015 \\
\hline Towards an internationalization of RDA management and development & Dunsire, G. & 2016 \\
\hline BIBFRAME transformation for enhanced discovery & Jin, Q.; Hahn, J.; Croll, G. & 2016 \\
\hline $\begin{array}{l}\text { Emerging information standards and technologies: cataloging and metadata } \\
\text { professionals'perspectives }\end{array}$ & Park, J. R.; Tosaka, Y. & 2017 \\
\hline FRBR: application of the model to textual documents & O'Neill, E.; Žumer, M. & 2018 \\
\hline
\end{tabular}

Fonte: Elaborado pelos autores (2019).

\section{Casos de uso identificados na Revisão Sistemática da Literatura}

Com a RSL foi possível analisar, estudar e compreender as tendências do domínio bibliográfico e sua aplicação em repositórios institucionais digitais. Ela propiciou a descoberta de exemplos práticos de aplicação, tanto do modelo conceitual FRBR quanto das orientações RDA em ambientes informacionais digitais, apontados como tecnologias computacionais e informacionais por estudos recuperados, como: Perfis de aplicação de metadados, BIBFRAME (Bibliographic Framework Initiative), LibFRBR, RIMMF (RDA In Many Metadata Formats).

\section{Perfis de aplicação de metadados}

Um dos principais exemplos abordados nos documentos analisados são os perfis de aplicação de metadados, mais especificamente de aplicação do padrão Dublin Core (DCAP, Dublin Core Application Profiles).

Coyle e Baker (2009) explicam que um DCAP é um documento (ou conjunto de documentos) que especifica e descreve os metadados usados em uma aplicação específica. Para isso, um DCAP: (a) descreve o que uma comunidade deseja realizar com sua aplicação (Requisitos Funcionais); (b) caracteriza os tipos de objetos descritos pelos metadados e seus relacionamentos (Modelo de Domínio); (c) enumera os termos de metadados a serem usados e as regras para seu uso (Perfil de Conjunto de Descrição e Diretrizes de Uso); e (d) define a sintaxe da máquina que será usada para codificar os dados (Diretrizes de Sintaxe e Formatos de Dados).

Percebe-se, portanto, que o desenvolvimento de perfis de aplicação de metadados, em especial voltados ao padrão Dublin Core, pode contribuir para uma melhor representação da informação. Quando específicos para trabalhos acadêmicos, esses perfis são chamados de Scholarly Works Application Profile (SWAP).

O detalhamento das características e das estruturas acerca dos perfis de aplicação de metadados identificados nessa pesquisa são encontrados nos estudos de Allinson (2008), de Chaudhri (2009) e de Solodovnik (2011), conforme mapeado na Revisão Sistemática da Literatura.

\section{Bibliographic Framework Initiative (BIBFRAME)}

Tendo em vista a ausência de garantia a respeito do atendimento, por parte do formato MARC 21, às exigências de descrição da informação na Web, a Library of Congress lançou, em 2011, o Bibliographic Framework 
Initiative (BIBFRAME). O intuito era apresentá-lo para consolidar o futuro da descrição bibliográfica na Web. (Silva et al., 2017).

O BIBFRAME é a base para o futuro da descrição bibliográfica que acontece na e como parte da Web e do atual mundo em rede. Foi projetado para se integrar e se envolver com a mais ampla comunidade de informações, além de atender às necessidades específicas de sua comunidade de manutenção. O BIBFRAME cumprirá tais propósitos, visando: (a) diferenciar claramente o conteúdo conceitual e suas manifestações físicas (por exemplo, obras e instâncias); (b) focar na identificação não ambígua.

De acordo com Arakaki et al. (2017), as tecnologias como o RDF (Resource Description Framework), o modelo conceitual FRBR e suas extensões, as diretrizes RDA, a linguagem XML (eXtensible Markup Language), entre outras, fazem parte do desenvolvimento do BIBFRAME, possibilitando ao modelo uma adequação às perspectivas e às tendências do tratamento da informação no cenário contemporâneo.

A literatura científica sobre a conceituação, as características e o contexto de desenvolvimento do BIBFRAME podem ser identificados e apontados nos estudos de Jin, Hahn e Croll (2016) e Park e Tosaka (2017).

\section{LibFRBR}

O LibFRBR é referenciado no trabalho de Chang et al. (2013) como uma ferramenta para aplicação usada para converter registros bibliográficos em estruturas FRBRizadas.

O LibFRBR desempenha um papel crucial no suporte aos sistemas baseados em FRBR, fornecendo uma interface FRBR para entrada e para armazenamento de dados. Esse software suporta funções para consulta, para saída e exibição de dados FRBR, além de possibilitar a conversão do formato MARC para o FRBR.

Nos estudos de Chang et al. (2013), o LibFRBR fora utilizado para a conversão de registros em formato FRBR no software Koha.

\section{Resource Description and Access in Many Metadata Formats}

O RIMMF (RDA In Many Metadata Formats) é um software livre, criado em 2012 por Deborah Fritz e Richard Fritz, para ser utilizado como uma ferramenta de treino para o processo de catalogação descritiva em formato RDA, em que o profissional catalogador possa visualizar e desenvolver registros descritivos projetados especificamente para o RDA. O RIMMF está em sua terceira versão (RIMMF3), de acordo com o site oficial do software (Fritz; Fritz, 2012).

O RIMMF também é concebido como uma ferramenta de visualização para entender os conceitos RDA, de atributos e de relacionamentos das entidades FRBR, o que permite a inserção de dados descritivos sobre os atributos de entidades FRBR relevantes e, em seguida, vincula os dados descritivos entre entidades relacionadas. Segundo apresentado nos trabalhos de Doyle (2015) e de Dunsire (2016), o RIMMF foi projetado para ilustrar algumas das muitas possibilidades de interface eventualmente disponíveis em um ambiente RDA/FRBR e pode ser potencialmente utilizado na construção e na modelagem dos ambientes informacionais digitais contemporâneos, como os repositórios institucionais digitais e os catálogos bibliográficos.

A análise do corpus documental dessa pesquisa permitiu a identificação de ferramentas, de aplicações, de diretrizes e de orientações em domínios heterogêneos, como o bibliográfico, o acadêmico e a Web. Isso possibilita um maior aproveitamento das tecnologias computacionais existentes, além de sua aplicação em ambientes informacionais específicos, como os repositórios institucionais digitais, preconizando os princípios de interoperabilidade entre ambientes e sistemas informacionais. 


\section{Conclusão}

Com o método de Revisão Sistemática da Literatura, foi possível traçar e analisar o estado da arte acerca da contribuição do domínio bibliográfico para os ambientes digitais, mais especificamente para os repositórios institucionais digitais. O estado da arte mapeou e identificou exemplos da aplicação de metadados baseados no modelo conceitual FRBR e nas orientações RDA em repositórios institucionais digitais, bem como casos de uso, entre os quais se destacam softwares, ferramentas, metodologias e tecnologias emergentes do domínio Web.

A busca e a recuperação de informações podem ser aprimoradas pelo modelo conceitual FRBR e pelas orientações RDA, pois, além de prover uma estrutura organizada dos atributos das entidades, o uso do conceito de entidade-relacionamento ajuda a relacioná-las de maneira ampla e precisa. Dessa forma, usuários podem realizar buscas eficientemente, encontrando objetos relacionados com maior facilidade.

Destaca-se que os registros pautados no padrão Dublin Core, como no caso dos repositórios institucionais, são caracterizados pela simplicidade e são passíveis de customização e de extensibilidade. Isso possibilita que as comunidades que o adotam possam adequá-lo às necessidades dos usuários. Além disso, promovem interoperabilidade e são reconhecidos e utilizados mundialmente (Souza; Bezerra; Costa, 2016).

A aplicação do modelo conceitual FRBR e das orientações RDA amplia a capacidade semântica dos sistemas de busca e aproxima-os das necessidades informacionais contemporâneas dos usuários no contexto tecnológico instaurado como a Web Semântica e o movimento Linked Data, contribuindo para uma melhor representação e para uma posterior recuperação da informação.

Compreender o domínio bibliográfico e sua potencialidade de aplicação em repositórios institucionais digitais apresenta-se como uma condição sine qua non para a identificação das tendências conceituais, metodológicas e tecnológicas do campo da Catalogação Descritiva. Além disso, essa compreensão traz contribuições para os estudos das formas de representação da informação e da modelagem de ambientes digitais mais adequadas e efetivas.

Um dos aspectos inovadores desta pesquisa se demonstra a partir do método utilizado para a prospecção da construção do arcabouço teórico e metodológico da Revisão Sistemática da Literatura, pouco conhecido, difundido e utilizado na comunidade de pesquisadores da Ciência da Informação, sobretudo no Brasil.

O estado da arte construído nesta pesquisa evidencia a contribuição deste estudo como uma fonte de informação em língua portuguesa na área da Catalogação Descritiva, pois apresenta as características, as etapas e os critérios rigorosos que garantem a qualidade e a reprodutibilidade dos documentos obtidos. Desse modo, oferece um referencial teórico e metodológico consistente e sistemático, requisitos indispensáveis para que a pesquisa realizada seja auditada, continuada e reutilizada sempre que necessário.

\section{Referências}

Allinson, J. Describing scholarly works with Dublin Core: a functional approach. Library Trends, n. 57, p. 221-243, 2008.

Alves, R. C. V. Metadados para representação e recuperação da informação em ambiente web. In: Seminário Serviços de Informação em Museus, 4., 2016, São Paulo. São Paulo: Pinacoteca de São Paulo, 2016, p. 95-106. Disponível em: http:// biblioteca.pinacoteca.org.br:9090/bases/biblioteca/11945. pdf\#page=95. Acesso em: 22 abr. 2020.

Arakaki, F. A. et al. BIBFRAME: tendência para a representação bibliográfica na web. Revista Brasileira de Biblioteconomia e
Documentação, v. 13, p. 2231-2249, 2017. Disponível em: https://rbbd.febab.org.br/rbbd/article/view/995/1030. Acesso em: 22 abr. 2020.

Assumpção, F. S.; Santos, P. L. V. A. C. A utilização do Resource Description and Access (RDA) na criação de registros de autoridade para pessoas, famílias e entidades coletivas. Encontros Bibli, v. 18, n. 37, p. 203-226, 2013.

Briner, R. B.; Denyer, D. Systematic review and evidence synthesis as a practice and scholarship tool. In: Rousseau, D. M. (ed.). Handbook of evidence-based management: 
companies, classrooms, and research. New York: Oxford University Press, 2012. p. 328-374. Available from: https:// www.oxfordhandbooks.com/view/10.1093/oxfordhb/9780 199763986.001.0001/oxfordhb-9780199763986-e-7. Access on: Apr. 222020

Castro, F. F.; Santos, P. L. V. A. C. A consistência da informação descritiva em repositórios digitais: caminhos para a interoperabilidade. In: Congresso Brasileiro de Biblioteconomia, Documentação e Ciência da Informação, 24., 2011, Maceió. Anais [...], Maceió, 2011. Disponível em: https://ri.ufs.br/ handle/123456789/345. Acesso em: 22 abr. 2020.

Cerrao, N. G. Análise das aplicações de metadados baseada em FRBR e RDA em repositórios institucionais digitais: contribuições do domínio bibliográfico. 2019. 122f. Dissertação (Mestrado em Ciência da Informação) - Universidade Federal de São Carlos, São Carlos, 2019.

Chang N. et al. Experimenting with implementing FRBR in a Chinese Koha system. Library Hi Tech News, v. 30, n. 10, p. 10-20, 2013. Available from: https://www.emerald.com/insight/ content/doi/10.1108/LHTN-09-2013-0054/full/html. Access on: Apr. 222020.

Chaudhri, T. Assessing FRBR in Dublin Core application profiles. Ariadne, n. 58, 2009. Available from: http://www. ariadne.ac.uk/issue/58/chaudhri/. Access on: Apr. 222020.

Coyle, K.; Baker, T. Guidelines for Dublin Core Application Profiles. United States: DCMI, 2009. Available from: http:// www.dublincore.org/specifications/dublin-core/profileguidelines/. Access on: Apr. 222020.

Doyle, H. FRBR, RDA and Law Libraries. Legal Information Management, v. 15, n. 4, p. 240-247, 2015.

Dunsire, G. Towards an internationalization of RDA management and development. Italian Journal of Library, Archives, and Information Science, v. 7, n. 2, 2016. Doi: https:// doi.org/10.4403/jlis.it-11708.

Fritz, D.; Fritz, R. RIMMF: RDA in Many Metadata Formats. Version 3. 2012 [software]. Available from: http://www. marcofquality.com/wiki/rimmf3/doku.php?id=rimmf. Acesso em: 22 abr. 2020.

International Federation of Library Associations and Institutions. Functional Requirements for Bibliographic Records: final report. München: K. G. Saur, 2009.
International Federation of Library Associations and Institutions. Statement of international cataloguing principles. Den Haag: IFLA, 2016. Available from: http://www.ifla.org/ publications/node/11015. Access on: Abr. 222020.

Jin, Q.; Hahn, J.; Croll, G. BIBFRAME transformation for enhanced discovery. Association for Library Collections and Technical Services, v. 60, n. 4, 2016. Available from: https://journals.ala. org/index.php/lrts/article/view/6134/7929. Access on: Apr. 222020.

Laboratório de Pesquisa em Engenharia de Software. StArt: State of the Art through Systematic Review. São Carlos: Ufscar, 2012. Disponível em: http://lapes.dc.ufscar.br/tools/start_ tool. [software]. Acesso em: 22 abr. 2020.

Moreno, F. P.; Arellano, M. A. M. Requisitos Funcionais para Registros Bibliográficos (FRBR): uma apresentação. Revista Digital de Biblioteconomia e Ciência da Informação, v. 3, n. 1, 2005. Disponível em: https://periodicos.sbu.unicamp.br/ojs/ index.php/rdbci/article/view/2052. Acesso em: 22 abr. 2020.

Oliver, C. Introdução à RDA: um guia básico. Brasília: Briquet de Lemos, 2011.

Park, J. R.; Tosaka, Y. Emerging information standards and technologies: cataloging and metadata professionals' perspectives. Library Hi Tech News, v. 34, n. 4, 2017. Doi: https:// doi.org/10.1108/LHTN-12-2016-0059.

RDA Steering Committee. Frequently asked questions. RDA Steering Committee, Chicago, 2018. Available from: http://rdarsc.org/content/rda_faq\#1. Access on: Dez. 312018.

Silva, L. C. et al. O código RDA e a iniciativa BIBFRAME: tendências da representação da informação no domínio bibliográfico. Em Questão, v. 23, n. 3, p. 131-157, 2017.

Solodovnik, I. Metadata issues in digital libraries: key concepts and perspectives. Italian Journal of Library, Archives and Information Science, v. 2, n. 2, 2011. Doi: https://doi. org/10.4403/jlis.it-4663.

Souza, E.; Bezerra, D.; Costa, W. Descrição de recursos em uma estrutura de metadados pautada no modelo FRBR. Em Questão, v. 22, n. 1, p. 113-136, 2016.

Tillett, B. What is FRBR?: a conceptual model for the bibliographic universe. Washington: Library of Congress, 2004. Available from: https://www.loc.gov/cds/downloads/FRBR.PDF. Acesso em: 22 abr. 2020 